\title{
Osteopetrosis in a Domestic Shorthair Cat
}

\author{
Rick Beishuizen ${ }^{1}$ Nermin Caliskan ${ }^{2}$ Andrea Gröne ${ }^{2}$ \\ Marianna A. Tryfonidou ${ }^{1}$ Björn P. Meij ${ }^{1}$ \\ ${ }^{1}$ Department of Clinical Sciences of Companion Animals, Utrecht \\ University Faculty of Veterinary Medicine, Utrecht, The Netherlands \\ 2 Department of Pathobiology, Utrecht University Faculty of \\ Veterinary Medicine, Utrecht, The Netherlands \\ ${ }^{3}$ Specialist Veterinary Clinic Utrecht, AniCura Group, Utrecht, \\ The Netherlands
}

VCOT Open 2021;4:e79-e85.

\author{
Susanne A.E.B. Boroffka ${ }^{3}$
}

\begin{abstract}
Address for correspondence Rick Beishuizen, DVM, Department of Clinical Sciences of Companion Animals, Utrecht University Faculty of Veterinary Medicine, Yalelaan 108, Utrecht 3508 TD, The Netherlands (e-mail: rickbeishuizen@gmail.com).
\end{abstract}

\begin{abstract}
Keywords

- cat

- osteopetrosis

- bone displasia

- inherited skeletal deformity

- pathologic fracture

The purpose of this case report was to describe a cat with generalized bone dysplasia, resembling osteopetrosis and Albers-Schönberg disease in humans. A 1-year-3-monthold, intact male, domestic shorthair cat had a 9-month history of multiple bone fractures without known trauma. Most fractures were treated conservatively and two by osteosynthesis. Bone healing occurred but recurring fractures eventually led to euthanasia. Radiographs, computed tomographic imaging, postmortem analysis and histopathologic examination revealed a generalized increase in bone density and mass with preservation of bone shape, obliteration of the bone marrow cavity and persistence of cartilage and primary trabeculae. Abuse and secondary bone diseases were excluded. History, diagnostic bloodwork, radiographs, computed tomographic imaging and histopathologic examination supported the diagnosis of inherited osteopetrosis and strongly resembled Albers-Schönberg disease in humans. The presence of osteoclasts suggested that the underlying pathology might be found in osteoclast dysfunction, deficient number of osteoclasts, inadequate recruitment of osteoclasts, or other micro environmental changes. In (young) cats that are presented with recurring fractures and the possible suspicion of abuse, inherited osteopetrosis should be considered as a differential diagnosis.
\end{abstract}

\section{Introduction}

Osteopetrosis is a heterogeneous group of acquired or hereditary bone dysplasias which are identified by osteosclerosis (increase in bone density and mass) without alteration of the overall bone shape. ${ }^{1}$ Osteopetrosis was first described over a century ago in humans as Albers-Schönberg disease. ${ }^{2}$ The condition has also been presumed in cats, ${ }^{3-15}$ but only the acquired form has been reported in a limited number of cases. The condition ranges in severity from asymptomatic to death at infancy and is caused by a failure of osteoclast

received

November 17, 2020

accepted after revision

December 9, 2020
DOI https://doi.org/

$10.1055 / \mathrm{s}-0040-1722735$.

ISSN 2625-2325. differentiation, function or mutation, resulting in impaired bone resorption and remodelling. ${ }^{4}$

In humans, there are multiple genetic subtypes. ${ }^{5}$ Autosomal dominant osteopetrosis (ADO) type 1 or ADO type 2 (also known as Albers-Schönberg) occurs at adolescence. Radiographs reveal generalized mild-to-moderate uniform osteosclerosis in the skull, spine and long bones (ADO type 1), sometimes together with a more multifocal hyperostosis causing a bone-withinbone appearance (ADO type 2$)^{1,5}$ Hanel and colleagues reported a 12-year-old cat with fibrosarcoma and osteopetrosis resembling Albers-Schönberg in humans. ${ }^{6}$ Autosomal recessive
(C) 2021. The Author(s).

This is an open access article published by Thieme under the terms of the Creative Commons Attribution License, permitting unrestricted use, distribution, and reproduction so long as the original work is properly cited. (https://creativecommons.org/licenses/by/4.0/)

Georg Thieme Verlag KG, Rüdigerstraße 14, 70469 Stuttgart, Germany 

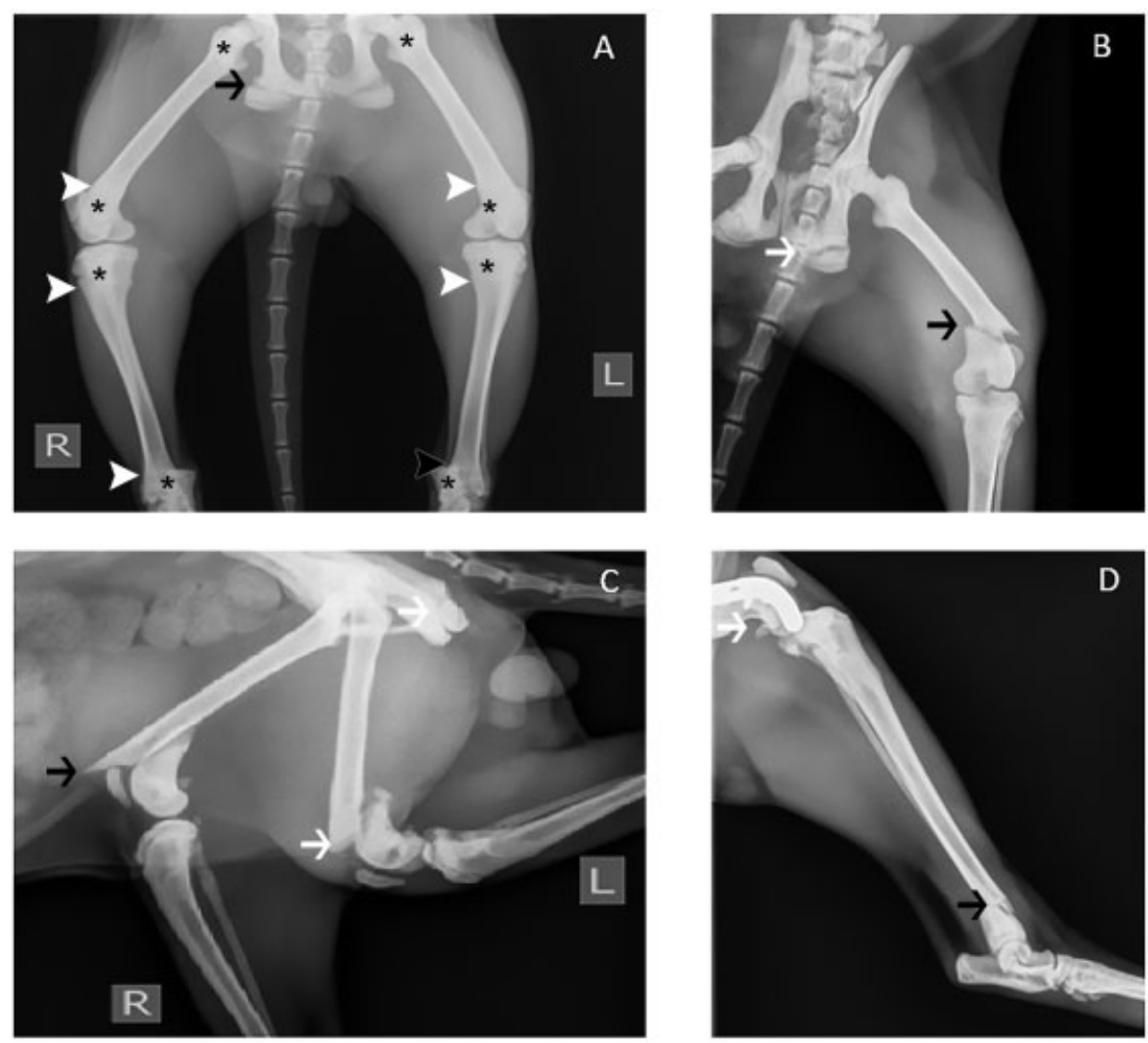

Fig. 1 Series of longitudinal radiographs of a cat with osteopetrosis that presented with recurrent lameness due to fractures of the ischium at age of 6 months (A), distal left femur at 8 months (B), distal right femur at 9 months (C) and left distal tibia/fibula at 11 months (D). The radiographic abnormalities noted at sequential times were increased opacity and width of metaphysis (black star), followed by a horizontal sclerotic line (white arrowhead), acute (sharp margins) fracture (black arrow) and eventually chronic fracture with consolidated callus formation (white arrow).

osteopetrosis (ARO) in humans is usually fatal at an early age (1,4,7 $^{1,7}$ An intermediate autosomal form becomes apparent at childhood and results in fractures and anemia. ${ }^{1,8}$ The last genetic subtype is a X-linked type which becomes apparent at infancy and consists of fractures and recurrent infections. ${ }^{8}$

Osteopetrosis can also be acquired, resulting from hypercalcitonism, prolonged vitamin D excess, systemic lupus erythematosus (SLE), renal failure, myeloproliferative disorders, leukaemia, viral infections and neoplasia. Wright and colleagues described 14 cats with acquired osteopetrosis, resulting from leukaemia (1), SLE-related illness (1), renal failure (1), neoplasia (3) and myeloproliferative disorders (8) and suggested to rename the condition to generalized endosteal hyperplasia because the described cases did not completely resemble osteopetrosis in humans. ${ }^{9}$ There is only one case report that suspects inherited osteopetrosis in a cat, but more likely was secondary, considering the old age of the cat (12 years) and the presence of a fibrosarcoma. ${ }^{6}$ The aim of the present case report is to describe a young adolescent cat with generalized bone dysplasia resembling inherited ADO in humans.

\section{Case Description}

A 1-year-3-month-old, intact male, domestic shorthair cat had a 9-month history of multiple bone fractures without known trauma. The cat had always been with the current owners and had been presented at a private veterinary clinic on 10 different occasions with sudden lameness, localized soft tissue swelling or painfulness. The cat was fully vaccinated, had regular unsupervised outdoor access until the age of 9 months and was fed with a commercially available feline diet. The cat had been presented at the age of 6 months with mild right hindlimb lameness and gingivitis, while the first deciduous teeth had fallen out. At the age of 8 months, a severe left hindlimb lameness became apparent. On radiographs, the distal and proximal metaphysis of both femora, tibia and fibula showed increased opacity and widening (-Fig. 1A). The pelvis showed an ill-defined transverse fracture of both tuber ischii. Both distal femora and proximal and distal tibiae showed a horizontal sclerotic line with proximal medial and lateral consolidated callus formation. The proximal left fibula showed a radiolucent line just distal of the growth plate, without dislocation. All distal metatarsal bones showed transverse fractures with periosteal new bone formation or increased opacity and thickening of the metaphysis. The owner opted for treatment of the fractures with external coaptating (splint) bandage. After 2 weeks, the cat was presented with severe lameness of the left hindlimb. On radiographic examination, the left distal femur showed an oblique transverse fracture at the horizontal sclerotic line noticed on earlier radiographs. The distal metaphysis of the 
Table 1 Results of haematology and blood biochemistry in a 1-year-old cat with osteopetrosis

\begin{tabular}{|c|c|c|}
\hline & Results & Reference range \\
\hline Haematocrit & $29 \%$ & $24-45 \%$ \\
\hline Haemoglobin & $9.0 \mathrm{~g} / \mathrm{dL}$ & $8-15 \mathrm{~g} / \mathrm{dL}$ \\
\hline Mean corpuscular haemoglobin concentration & $31 \mathrm{~g} / \mathrm{dL}$ & $30.0-36.9 \mathrm{~g} / \mathrm{dL}$ \\
\hline White blood cell count & $15.3 \times 10^{9} / \mathrm{L}$ & $5.0-18.9 \times 10^{9} / \mathrm{L}$ \\
\hline Granulocytes & $10.7 \times 10^{9} / \mathrm{L}$ & $2.5-12.5 \times 10^{9} / \mathrm{L}$ \\
\hline Lymphocytes/monocytes & $4.6 \times 10^{9} / \mathrm{L}$ & $1.5-7.8 \times 10^{9} / \mathrm{L}$ \\
\hline Platelets & $263 \mathrm{~K} / \mu \mathrm{L}$ & $175-500 \mathrm{~K} / \mu \mathrm{L}$ \\
\hline Glucose & $7.28 \mathrm{mmol} / \mathrm{L}$ & $4.28-8.50 \mathrm{mmol} / \mathrm{L}$ \\
\hline Urea & $10.7 \mathrm{mmol} / \mathrm{L}$ & $5.7-11.8 \mathrm{mmol} / \mathrm{L}$ \\
\hline Creatinine & $80 \mu \mathrm{mol} / \mathrm{L}$ & $53-141 \mu \mathrm{mol} / \mathrm{L}$ \\
\hline Phosphate & $1.71 \mathrm{mmol} / \mathrm{L}$ & $1.45-3.35 \mathrm{mmol} / \mathrm{L}$ \\
\hline Calcium & $2.25 \mathrm{mmol} / \mathrm{L}$ & $1.98-2.83 \mathrm{mmol} / \mathrm{L}$ \\
\hline Total protein & $69 \mathrm{~g} / \mathrm{L}$ & $52-82 \mathrm{~g} / \mathrm{L}$ \\
\hline Albumin & $27 \mathrm{~g} / \mathrm{L}$ & $22-39 \mathrm{~g} / \mathrm{L}$ \\
\hline $\mathrm{pH}^{\mathrm{a}}$ & 7.43 & $7.24-7.40$ \\
\hline Partial carbon dioxide pressure & $36 \mathrm{~mm} \mathrm{Hg}$ & $34-38 \mathrm{~mm} \mathrm{Hg}$ \\
\hline Bicarbonate & $22,5 \mathrm{mmol} / \mathrm{L}$ & $22-24 \mathrm{mmol} / \mathrm{L}$ \\
\hline Anion gap & $20,3 \mathrm{mmol} / \mathrm{L}$ & $13-27 \mathrm{mmol} / \mathrm{L}$ \\
\hline Total carbon dioxide $^{a}$ & $23.6 \mathrm{mmol} / \mathrm{L} \downarrow$ & $27-31 \mathrm{mmol} / \mathrm{L}$ \\
\hline Sodium & $155 \mathrm{mmol} / \mathrm{L}$ & $150-165 \mathrm{mmol} / \mathrm{L}$ \\
\hline Potassium $^{a}$ & $3.3 \mathrm{mmol} / \mathrm{L} \downarrow$ & $3.5-5.8 \mathrm{mmol} / \mathrm{L}$ \\
\hline Chloride & $115 \mathrm{mmol} / \mathrm{L}$ & $112-129 \mathrm{mmol} / \mathrm{L}$ \\
\hline Globulins & $42 \mathrm{~g} / \mathrm{L}$ & $28-48 \mathrm{~g} / \mathrm{L}$ \\
\hline Alanine aminotransferase & $79 \mathrm{U} / \mathrm{L}$ & $12-115 \mathrm{U} / \mathrm{L}$ \\
\hline Alkaline phosphatase & $25 \mathrm{U} / \mathrm{L}$ & 14-192 U/L \\
\hline Total bilirubin & $2 \mu \mathrm{mol} / \mathrm{L}$ & $0-15 \mu \mathrm{mol} / \mathrm{L}$ \\
\hline Cholesterol & $3.81 \mathrm{mmol} / \mathrm{L}$ & $1.6-4.94 \mathrm{mmol} / \mathrm{L}$ \\
\hline Amylase & $605 \mathrm{U} / \mathrm{L}$ & $500-1400 \mathrm{U} / \mathrm{L}$ \\
\hline
\end{tabular}

${ }^{a}$ Values depicted in bold are outside the reference range.

right femur and right and left tibia and fibula (-Fig. 1B) showed increased opacity and width of the bone. All distal metatarsal bones showed transverse sclerotic fracture lines with slight consolidation and bridging callus formation. At the age of 9 months, the cat was presented with severe lameness of the right hindlimb. Radiographs revealed an oblique transverse fracture of the right distal femur at the level of the horizontal sclerotic line noted on earlier radiographs (-Fig. 1C). Blood biochemistry and haematology were performed. Apart from slight hypokalaemic alkalosis with decreased carbon dioxide, all blood values were within reference range and revealed no underlying metabolic disorder ( - Table 1). Both distal femoral fractures were treated by open reduction and internal fixation.

At the age of 14 months, the cat was presented again with severe left hindlimb lameness. Mediolateral radiographs showed an oblique transverse fracture of the tibia and fibula.
The distal left femur with the osteosynthesis plate showed consolidated fracture healing and callus formation ( - Fig. 1D). The tibia fracture was treated with an external coaptating splint. After 4 weeks, the cat suddenly became lame again on his left hindlimb while playing with a toy.

The Academic Hospital in Utrecht was then consulted to review the case because fractures due to abuse were considered as a possibility. Radiographs were made of both front and hind limbs, thorax and skull. The left distal radius and ulna showed a vague transverse irregular fracture without dislocation. The right distal ulna showed a vague irregular fracture without dislocation but with possibly small fragments and rounded consolidating callus formation. At least two phalanges of the left hindlimb had fractures with mild dislocation distally. The left distal tibia and fibula had an oblique transverse fracture and cranial and caudal callus formation. The distal left femur showed further consolidating callus 

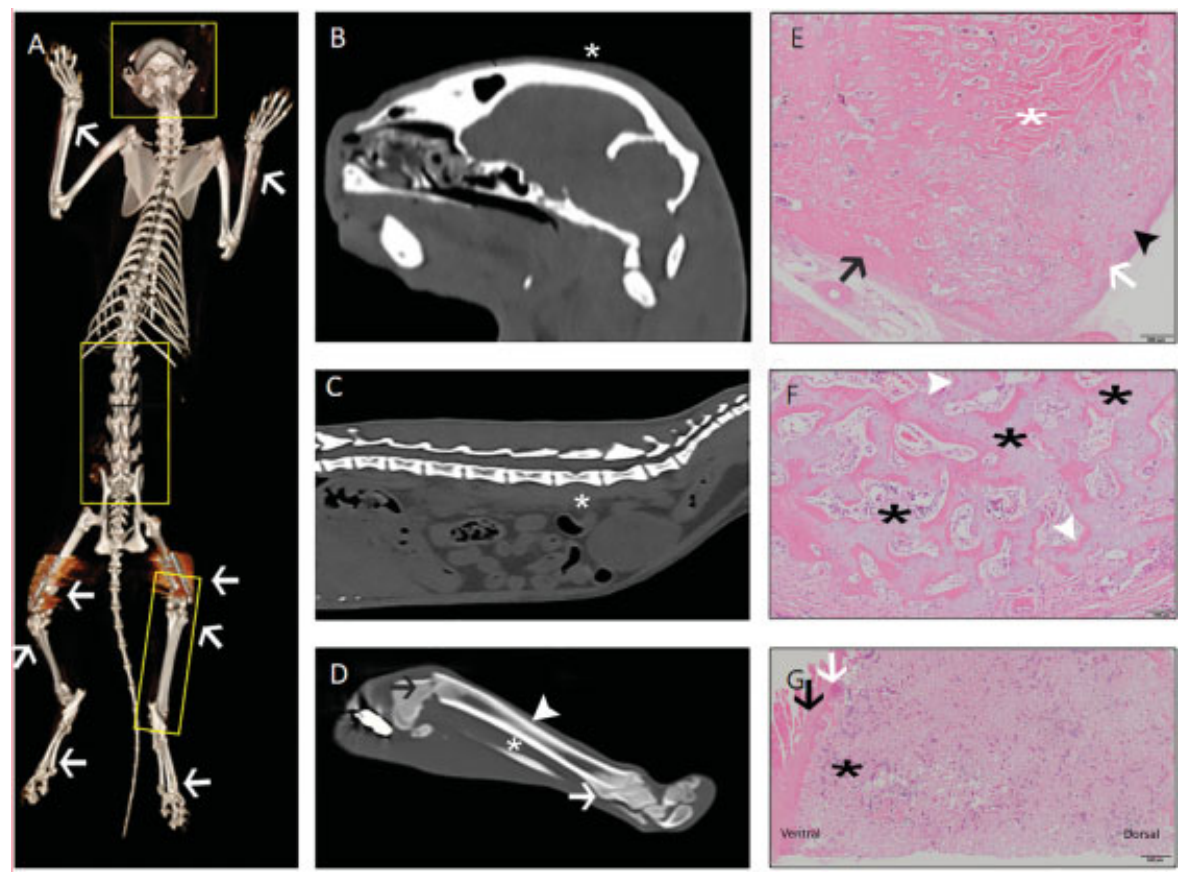

Fig. 2 Three-dimensional reconstruction (A) of post-mortem computed tomography of a cat with osteopetrosis revealing multiple fractures (arrows). The cat was 1 year and 3 months old. Sagittal multiplanar reconstruction of the skull (B), lumbar spine (C) and left tibia (D) showing increase in bone density and mass (white star) with narrowing of the medullary cavity (white arrowhead), acute (black arrow) and chronic fracture with callus formation (white arrow). Haematoxylin and eosin staining of the left tibia (E, F), with respectively, magnification $x 40$ $(\mathrm{bar}=500 \mu \mathrm{m})$ and $\times 200(\mathrm{bar}=100 \mu \mathrm{m})$, and lumbar vertebrae $(G)$, magnification $\times 40(\mathrm{bar}=500 \mu \mathrm{m})$, of a cat with osteopetrosis. The left tibia section (E) was taken from the transition of distal diaphysis to metaphysis. The cortex is noted as a zone of compact dense bone (black arrow). There are interconnecting variably thick bands of lamellar bone (white asterisk), causing obliteration of the marrow cavity and loss of bone marrow (white arrow). The thickened cortical bone is partially surrounded by a sub-periosteal cartilaginous cap (black arrowhead). Prominent retention of cartilage is visible (black asterisk) in a close-up through the metaphysis of the left tibia (F), where there is attachment of the joint capsule and periosteum. The frequent scalloped appearance (white arrowhead) of the edges of the bony trabecula suggests bony remodelling, although on haematoxylin and eosin staining, osteoclasts are not easily identified in any of these sections. In an overview of a lumbar vertebral body $(\mathbf{G})$ the ventral and dorsal sides are depicted, the periosteum is noted by the black arrow. The eosinophilic line represents the cortex (white arrow). Multifocally to coalescing, the medullary cavity is obliterated due to interconnecting variably thick bands of lamellar bone with only few remaining islands of hematopoietic cells (black asterisk). The endplates are not captured on this photomicrograph.

formation. On the distal right tibia, a horizontal sclerotic line was present with slight widening of the metaphysis. Mandibula and maxilla showed increased medullary radio-opacity. In the dorsoventral view, the mandibula appeared widened. The dorsal cranium appeared thickened and sclerotic. All permanent dental elements were present but below the gingiva. The incisor and premolar deciduous teeth were still present.

At request of the owner, the cat was euthanatized and with the owner's consent postmortem examination was conducted. On the cadaver, total body computed tomography was performed with a third-generation single slice helical computed tomography (CT) scanner (Philips Secura, Philips $\mathrm{NV}$, Eindhoven, The Netherlands) with a $1 \mathrm{~mm}$ slice thickness, 65-99 mA, 100kV, WL: $600 \mathrm{WW}: 3000$ (-Fig. 2A). The CT scan was followed by necropsy; for bone histology, the right ulna, left tibia, lumbar vertebrae, ribs and skull were fixated in $4 \%$ phosphate-buffered formalin for at least 48 hours; tissues were then decalcified in $10 \%$ ethylene diamine tetra-acetic acid and embedded in paraffin. Four $\mu \mathrm{m}$ thick sections were stained with haematoxylin and eosin (H\&E) and tartrate-resistant acid phosphatase to evaluate the presence of osteoclasts. Liver and kidney tissue, also embedded in paraffin, was tested with polymerase chain reaction (PCR) for the presence of feline leukaemia virus (FeLV).

The CT confirmed the thickened and sclerotic dorsal skull bone ( - Fig. 2B) and the permanent dental elements that had not erupted. The vertebrae were increased in attenuation, indicating thickening of the compacta ( $\mathbf{- F i g}$. $\mathbf{2 C}$ ). The front limbs contained chronic and acute fractures in the left distal radius and ulna and in the right distal ulna. Increased attenuation and thickening were present in the proximal and distal metaphysis of the right and left humerus and surrounding increased soft tissue attenuation was noted. The ribs and pelvis showed increased attenuation and chronic fractures were present in the left and right os ischium of the pelvis. The hindlimbs showed chronic and acute fractures in both distal femurs, tibias (-Fig. 2D), fibulas, all metatarsal bones and two phalanges I. Hyperattenuation and thickening were present in the proximal and distal metaphysis of both femurs and were surrounded by increased soft tissue attenuation.

Necropsy revealed that the cat had normal body fat reserves. The ribs were brittle. The distal portion of both 
the right ulna and left tibia had a slightly irregular thickening of the bone. The trachea, lungs, heart, liver, spleen and kidneys were unremarkable, except for abnormalities that were attributed to euthanasia. The liver did not reveal evidence of extramedullary haematopoiesis. Findings in other organs, including parathyroid glands, cerebrum and cerebellum, were unremarkable.

After decalcification, further microscopic evaluation of transverse sections of the right ulna, left tibia and ribs revealed massive thickening of the cortices with increased bone density (osteosclerosis) and retention of primary trabeculae at the transition of the cortex to the medulla in the tibia ( - Fig. 2E) at the level of the diaphysis. Within the tibia, the retained primary trabeculae extended to the transition of diaphysis to metaphysis. The thickened cortical bone (compaction) was partially surrounded by a subperiosteal cartilaginous cap, consisting of interconnecting variably thick bands of lamellar bone admixed with woven bone ('bone in bone' appearance) with a variable cartilage component (retention of cartilage). Due to this closely interdigitating and packed pattern, which almost completely obliterated the marrow cavity, there was only a minimal amount of bone marrow visible ( - Fig. 2 E and $\mathbf{G}$ ). Haematopoietic cells were multifocally present between the trabeculae. There was marked retention of cartilage in the metaphysis of the tibia, at the level of attachment of the joint capsule and periosteum (-Fig. 2F). Growth plate was not captured in any of the examined histological slides. The lumbar vertebral body showed multifocal persistent cartilage and primary trabeculae, but compaction of the cortex was not readily evident (-Fig. 2G). However, prominent narrowing of the medullary cavity did correlate to the radiographic alterations in this region. The skull showed a multifocal to almost diffuse increase of cortical bone. There was no evidence of inflammation, nor were there changes suggestive of an underlying viral condition. Osteoclasts were not obviously present in any of the examined decalcified H\&E-stained sections, although many trabeculae showed a scalloped outline, which was suggestive of bony remodelling (-Fig. 2F). Therefore, an additional staining to identify osteoclasts was performed. Sections of the left tibia and lumbar vertebrae revealed tartrate-resistant acid phosphatase positive multinucleated cells, indicative of osteoclasts (-Fig. 3). No evident Howship's lacunae were noted in these sections. The PCR for the presence of FeLV was negative.

\section{Discussion}

This case report describes a pre-adolescent cat presented with multiple chronic and acute spontaneous fractures over a period of 9 months that raised the suspicion of abuse. Other possible causes included inherited osteopetrosis and less likely secondary bone diseases. ${ }^{1,4}$ Radiographs, CT imaging and microscopic histopathologic examination revealed a generalized increase in bone density and mass without alteration of overall bone shape but with obliteration of the bone marrow cavity. Found were also persistence of non-resorbed calcified cartilage and primary trabeculae,

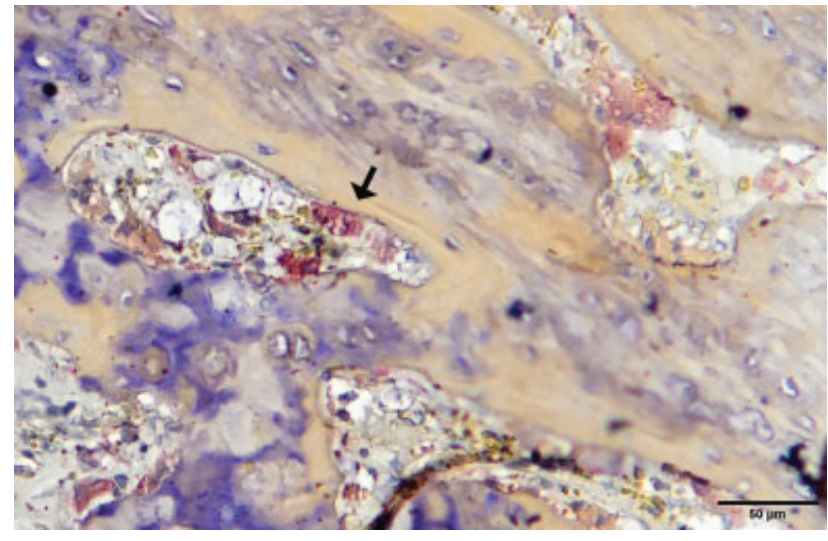

Fig. 3 Tartrate-resistant acid phosphatase staining of a bone section from a cat with osteopetrosis revealing positive multinucleated cells, indicative of osteoclasts (black arrow). No evident Howhip's lacunae were noted. Magnification x400 (bar $=50 \mu \mathrm{m})$.

presence of multiple acute and chronic fractures with signs of callus formation and dental impaction. These findings are consistent with inherited or acquired osteopetrosis.

Diagnostic bloodwork showed no abnormalities that could indicate secondary bone disease. Anaemia or leukopenia was absent. Furthermore, there were no indications for liver or kidney disease. The slight hypokalaemic alkalosis with decreased carbon dioxide levels could be explained with hyperventilation due to pain and stress. ${ }^{10}$ Secondary osteopetrosis has been reported in cats after inoculation with FeLV, but in our case a PCR for FeLV was negative. ${ }^{11}$ Although the test result might be a false negative, both FeLV and FIV were also unlikely considering the young age of the cat and the absence of FIVrelated or FeLV-related blood-abnormalities. ${ }^{12,13}$ Taken altogether, the haematologic values, as well as the history, physical examination, diagnostic imaging and histopathological examination excluded secondary bone disease.

Animals with multiple chronic and/or acute idiopathic fractures fit the clinical presentation of osteopetrosis but should also raise the suspicion of abuse. Abused patients usually present with fractures located in the areas of skull, ribs, teeth and vertebrae and often coincide with other injuries like bruising, burns, oedema, lacerations and broken claws. ${ }^{14}$ Abuse was considered unlikely in our case because fractures were present in different locations, the bones revealed an underlying pathology, there were no other injuries and fractures occurred in locations where, on previous radiographs, osteosclerotic lines were seen.

There are multiple forms of inherited osteopetrosis. Classic and neuropathic ARO are less likely to be the underlying pathology in this cat because of the lack of brain abnormalities, hypocalcaemia and bone marrow failure. ${ }^{1,4}$ The ARO with renal tubular acidosis seems improbable based on the lack of renal tubular acidosis, any intracranial calcifications or compressive neuropathy. Although urine was not available for examination, there was no hyperchloraemic metabolic acidosis present. Brain calcifications were not observed on $\mathrm{H} \& \mathrm{E}$ staining nor on CT images of the skull. Although CT is the most sensitive imaging modality for calcifications in brain 
tissue, mineralization's smaller than $1.5 \mathrm{~mm}$ might not be detected. $^{15}$ The lack of brain abnormalities, anaemia and renal tubular acidosis also rules out the intermediate autosomal form of osteopetrosis. ${ }^{1,4}$ The absence of recurrent infections, lymphedema and anhidrotic ectodermal dysplasia excludes the rare X-linked type of osteopetrosis. ${ }^{1}$ The ADO type 1 is unlikely since patients do not present with fractures. 1

Considering the differential diagnosis and clinical, biochemical, radiographical and histopathological findings, this case strongly resembles ADO type 2, also known as AlbersSchönberg disease. ${ }^{1}$ This case was also presented at adolescence with diffuse pain on skeletal palpation, fractures and impacted teeth. Radiographs in our cat revealed a generalized mild-to-moderate uniform osteosclerosis, which is also seen in ADO Type 2. It should be noted that Albers-Schönberg disease is a very heterogeneous condition in humans, resulting in a wide variety of clinical presentations. ${ }^{16}$ Presumably, the same may occur in cats. In comparison, dogs that were reported with osteopetrosis presented at a very young age with generalized osteosclerosis, fractures and non-regenerative anaemia due to bone filled marrow cavities. In dogs, osteopetrosis seems to resemble ARO or intermediate autosomal osteopetrosis. ${ }^{17,18}$

The mutation causing osteopetrosis in cats remains to be determined. In humans, mutations in at least ten genes cause the various types of osteopetrosis and lead to osteoclast autonomous defects or micro environmental changes. ${ }^{16}$ These changes affect the maturation ability of osteoclast progenitors and/or their ability to resorb bone. ${ }^{4,16}$ Mature osteoclasts dissolve bone mineral and degrade bone matrix, while new bone is deposited by osteoblasts, in the process of bone remodelling, a process that continuously takes place after birth during skeletal growth. ${ }^{4}$ Osteoclast dysfunction will lead to an accumulation of bone within a defective architecture, making the bones brittle and susceptible to fracture. Most of the causative mutations encode proteins involved in regulating the intra- and extracellular $\mathrm{pH}$ of osteoclasts. ${ }^{16,19,20}$ An osteoclast dysfunction through mutation in the CLCN7 gene or PLEHKM1 gene will result in autosomal recessive osteopetrosis. Dominant negative mutations in the CLCN7 gene, encoding the chloride channel coupled to the osteoclast $\mathrm{H}^{+}$-ATPase in the ruffled border, are responsible for $\sim 75 \%$ of human cases of ADO, 10 to $15 \%$ of cases of ARO and of all known cases of intermediate autosomal osteopetrosis in man. ${ }^{4,21}$ In a CLCN7 knockout mice model, osteoclasts were present but were unable to acidify the resorption lacunae, resulting in severe osteopetrosis. ${ }^{22}$ Missense mutations in PLEHKM1, a protein involved in vesicle trafficking and acidification, result in altered bone resorption and have also been implicated in Albers-Schönberg disease in man. In $\sim 30 \%$ of all cases of human osteopetrosis, the cause of the condition remains unknown. In the here presented case, tartrate-resistant acid phosphatasepositive osteoclasts were identified in tissue sections where fracture healing processes were ongoing, suggesting a loss of function mutation. Unfortunately, there is no reliable genetic material available from the present case or his siblings to test genes possibly involved. No information was available of the parents or siblings regarding similar disorders. In future cases, it would be interesting to sequence the whole genome or the feline equivalents of the mutation sites known in human.

In conclusion, this report describes the first case of a cat with inherited osteopetrosis resembling the human AlbersSchönberg disease. The presence of osteoclasts suggests that the underlying pathology might be found in osteoclast dysfunction, just as in Albers-Schönberg disease in humans. Inherited osteopetrosis should be considered as a differential diagnosis in (young) cats that are presented with recurring, multiple, pathologic or idiopathic fractures.

\section{Conflict of Interest}

None declared.

\section{References}

1 Van Hul W, Vanhoenacker F, Balemans W, Janssens K, De Schepper AM. Molecular and radiological diagnosis of sclerosing bone dysplasias. Eur J Radiol 2001;40(03):198-207

2 Albers-Schönberg HE. Radiographic images of a rare bone disease. Munch Med Wochenschr 1904;(51):365-368

3 Fujita M, Takaishi Y, Nagae H, et al. Osteopetrosis-like disease in a cat with respiratory distress. J Vet Med Sci 2007;69(06):687-690

4 Stark Z, Savarirayan R. Osteopetrosis. Orphanet J Rare Dis 2009;4 (05):5

5 Shapiro F. Osteopetrosis. Current clinical considerations. Clin Orthop Relat Res 1993;(294):34-44

6 Hanel RM, Graham JP, Levy JK, Buergelt CD, Creamer J. Generalized osteosclerosis in a cat. Vet Radiol Ultrasound 2004;45(04): 318-324

7 Sobacchi C, Schulz A, Coxon FP, Villa A, Helfrich MH. Osteopetrosis: genetics, treatment and new insights into osteoclast function. Nat Rev Endocrinol 2013;9(09):522-536

8 Van Hul W. Sclerosing bone disorders: a lot of knowns but still some unknowns. Bonekey Rep 2012;1(06):97

9 Wright MW, Hudson JA, Hathcock JT. Osteopetrosis in cats: clarification of a misnomer. Vet Radiol Ultrasound 2003;(44):106

10 Kaneko JJ, Harvey JW, Bruss ML, Carlson GP, Bruss M. Fluid, electrolyte, and acid-base balance. In: Clinical Biochemistry of Domestic Animals. Cambridge, MA: Academic Press; 2008:529-559

11 Hoover EA, Kociba GJ. Bone lesions in cats with anemia induced by feline leukemia virus. J Natl Cancer Inst 1974;53(05):1277-1284

12 Gleich S, Hartmann K. Hematology and serum biochemistry of feline immunodeficiency virus-infected and feline leukemia virus-infected cats. J Vet Intern Med 2009;23(03):552-558

13 Gleich SE, Krieger S, Hartmann K. Prevalence of feline immunodeficiency virus and feline leukaemia virus among client-owned cats and risk factors for infection in Germany. J Feline Med Surg 2009;11(12):985-992

14 Intarapanich NP, McCobb EC, Reisman RW, Rozanski EA, Intarapanich PP. Characterization and comparison of injuries caused by accidental and non-accidental blunt force trauma in dogs and cats. J Forensic Sci 2016;61(04):993-999

15 Tsuruda JS, Bradley WG. MR detection of intracranial calcification: a phantom study. AJNR Am J Neuroradiol 1987;8(06): 1049-1055

16 Del Fattore A, Cappariello A, Teti A. Genetics, pathogenesis and complications of osteopetrosis. Bone 2008;42(01):19-29

17 Lees GE, Sautter JH. Anemia and osteopetrosis in a dog. J Am Vet Med Assoc 1979;175(08):820-824

18 Riser WH, Fankhauser R. Osteopetrosis in the dog: a report of three cases. Vet Radiol. 1970;11(01):29-34 
19 Thomson RG. Failure of bone resorption in a calf. Pathol Vet 1966; 3(03):234-246

20 Michou L, Brown JP. Genetics of bone diseases: Paget's disease, fibrous dysplasia, osteopetrosis, and osteogenesis imperfecta. Joint Bone Spine 2011;78(03):252-258
21 Tolar J, Teitelbaum SL, Orchard PJ. Osteopetrosis. N Engl J Med 2004;351(27):2839-2849

22 Kornak U, Kasper D, Bösl MR, et al. Loss of the ClC-7 chloride channel leads to osteopetrosis in mice and man. Cell 2001;104 (02):205-215 